\title{
Educational Use of the ARGESIM Benchmarks for Modelling Approaches and Simulation Implementations
}

\author{
Felix Breitenecker ${ }^{1 *}$, Andreas Körner ${ }^{1}$, Horst Ecker ${ }^{2}$ \\ ${ }^{1}$ Mathematical Modelling and Simulation Group, Inst. of Analysis and Scientific Computing, ${ }^{2}$ Inst. of Mechanics \\ and Mechatronics; TU Wien, Wiedner Hauptstrasse 8-10, 1040 Vienna, Austria; felix.breitenecker@tuwien.ac.at
}

SNE 28(4), 2018, 215 - 218, DOI: 10.11128/sne.28.bne.10453

Received: November 15, 2018;

Accepted: November 30, 2018

SNE - Simulation Notes Europe, ARGESIM Publisher Vienna ISSN Print 2305-9974, Online 2306-0271, www.sne-journal.org

Abstract. This short note in SNE 28(4) sketches educational aspects of the 'ARGESIM Benchmarks for Modelling Approaches and Simulation Implementations' published since 1990 in SNE. Interestingly, it turned out, that along with the various benchmark publications in SNE, the benchmarks are used as examples, case studies, and lab work in simulation education in academia. Indeed, most of the benchmarks are a very useful basis for education in modelling and simulation. This contribution lists shortly the educational benefits of each benchmark - a detailed investigation of the educational aspects of the benchmarks, together with a description of classification and development of the benchmarks is scheduled for SNE 29 (1), March 2019.

\section{Introduction}

The ARGESIM Benchmarks for Modelling Approaches and Simulation Implementations are a success story in the simulation community. With up to now 24 benchmarks defined, and 354 benchmark solutions, benchmark reports, or benchmark studies published in SNE - Simulation Notes Europe, the scientific membership journal of EUROSIM, the Federation of European Simulation Societies. The benchmark publications have become a forum for information exchange on modelling approaches, simulation implementations, and features of simulation systems.

Interestingly, it turned out, that along with the various benchmark publications in SNE, the benchmarks are used as examples, case studies, and lab work in simulation education in academia. Indeed, most of the benchmarks are a very useful basis for education in modelling and simulation, at modelling level, and at implementation level.
The benchmarks may also be used for education in application areas, as introductory case studies in mechanical engineering, electrical engineering, economics, biology, etc.

This contribution presents shortly the publication possibilities for benchmark publications generally and for education-oriented benchmark publications (Section 1) and lists the educational benefits of each of the 24 benchmarks (Section 2). The last section comments the future development of new benchmark definitions and benchmark solutions to be initiated.

A detailed investigation of the educational aspects of the benchmarks, together with a description of a classification helpful for education and the development of the benchmarks is scheduled for SNE 29 (1), March 2019 [1].

\section{Benchmark Publications}

ARGESIM, a non-profit organisation for information and publication on simulation, started in 1990 the series $A R$ GESIM Comparisons of Simulation Software with modelling and simulation tasks based on relatively simple, easily comprehensible processes. In the beginning, simulationists were invited to prepare a comparison solution to be published in SNE as one-page solution. Along with development of system simulation, also the Comparisons of Simulation Software developed further on towards the ARGESIM Benchmarks for Modelling Approaches and Simulation Implementations.

In 2006 and in 2018, re-organisations of the publication strategy improved the publication possibilities for benchmark publications, including benchmark publications with emphasis on education:

- Extended definitions of new benchmarks:

SNE introduces new benchmarks with extended scope and indication of educational aspects, e.g. comparing modelling and simulation paradigms, dealing with more complex experiments, and formulating models and tasks especially for educational use - indicating educational aspects, as with benchmarks C19, C20, C21 and planned benchmark C22. 
- Revised definitions of previous benchmarks:

SNE is publishing revised definitions of previous comparisons, updating models, extending the scope, and underlining the educational aspects - in order to continue them as new benchmark also for benchmark contributions with emphasis on education, e.g. C12.

The benchmark publications now may have different content, structure, emphasis, and length - and the authors may underline educational aspects, or may shape the benchmark contribution for use in education. Modellers, simulationists, and lecturers in modelling and simulation are invited to take the challenge to prepare, realise and submit a

- Benchmark Solution with concise description of model implementation and experimentation tasks (two pages SNE), or a

- Benchmark Report with sufficient detailed description of model implementation with variants and adequate experiment formulations (four to six pages SNE), or an

- Educational Benchmark Report, a benchmark report with educational aspects or generally addressing use in education (four to six pages SNE), or a

- Benchmark Study presenting e.g. different / alternative / comparative modelling approaches and sketching analysis variants or supplemental model experiments (six to ten pages SNE), or an

- Educational Benchmark Study, a benchmark study for use in education, or at least with essential educational aspects (six to twelve pages SNE) - see e.g. contribution [2] in this SNE.

\section{Benchmarks for Education}

In the following the up to now defined comparisons and benchmarks are briefly investigated with respect to their suitability for educational purposes:

- C1 Lithium-Cluster Dynamics, SNE 0(1), 1990, is based on three coupled stiff ODEs describing a process in surface physics. Simulation beginners can learn to deal with stiff systems, to perform logarithmic parameter variations, and calculate steady states - using a simulation tool or a calculation tool.

- C2 Flexible Assembly System, SNE 1(1), 1991, is an introductory case study for process modelling with six process station around a circular transport belt for pallets. Novices get an introduction into process modelling and simulation, using an appropriate process simulation tool.
- C3 Generalized Class-E Amplifier, SNE 1(2), 1991, describes an RLC circuit with time-varying resistor. The case study is suited for introduction into analogue circuit modelling - by means of general or application-oriented simulation tools.

- C4 Dining Philosophers I, SNE 1(3), 1991, and C10 Dining Philosophers II, SNE 6(3), 1996,

This almost classical process describing the competition for limited resources is a typical case study for education. While $\mathrm{C} 4$ is very generally defined and invites for analysis of any kind, C10 is defined with emphasis on time domain analysis. The benchmarks are very well suited for education, using arbitrary calculation tools.

- C5 Two State Model, SNE 2(1), 1992, is based on two linear ODEs with state-dependent switching parameters and tests state event detection not really suited for education.

- C6 Emergency Department SNE 2(3), 1992, is based on a process model for follow-up treatment in a hospital's emergency department - with real-world data. Novices get an introduction into process modelling and simulation in a health care system, and health care people get an introduction to simulation capabilities - by means of an appropriate process simulation tool.

- C7 Constrained Pendulum, SNE 3(1), 1993, describes the classical pendulum, which hits a pin and continues swinging with switching states. Beginners in simulation can study the handling of simple state events, using an appropriate simulation or also a general calculation tool.

- C8 Canal-and-Lock System, SNE 6(1), 1996, is based on the scheduling of barges moving through a lock. The benchmark mainly tests features of discrete simulators for modelling complex logic, to validate logic through use of deterministic data, and to check for variance-reduction capabilities. The case study may be of educational interest for process modelling, but also for training programming of scheduling - using a process simulation tool, or direct programming.

- C9 Fuzzy Control of a Two Tank System, SNE 6(2), 1996, revised SNE 16(3), 2006, investigates the efficiency of fuzzy control for a simple hydraulic system. This benchmark may be of interest for introduction into fuzzy control, and simulation beginners learn to implement fuzzy structures by means of simulation tools with (easy) or without (laborious) fuzzy control features, or by classical calculation tools (challenging). 
- C11 SCARA Robot, SNE 8(1), 1998, makes use of the implicit model of a 3DOF robot with classic control and control for collision prevention. This benchmark is very suitable for education purposes. Beginners and trained simulationists can learn about implicit equation-base modelling or about physical power-based block-oriented modelling, and students from mechanical engineering get an introduction to mechanical modelling and simulation. Software tools for education range from multibody systems via system simulators to calculation tools.

- C12 Collision of Spheres, SNE 9(3), 1999, allows different approaches for the analysis of movement and for collision of spheres in a row, investigating the impact from elastic to plastic collisions. This benchmark addresses not only system simulation, it is a classical mechanical or physical case study mainly suited for use in education. Students in technical science can learn about collision phenomena, and novices in simulation get knowledge in state event handling. Interestingly, a broad variety of software tools can be used, from physical simulators to spreadsheet tools.

\section{- C13 Crane Crab and Embedded Control,} SNE 11(1), 2001; rev. SNE 17(1), 2007,

is based on modelling and digital control of a crane crab. The discrete control is designed by means of a state space observer, and by state space control. The revised definition of this benchmark outlines the educational aspects: for simulationists, comparison of models, investigation of discrete control elements, and state-dependent security interventions; and for students in control engineering, modelling and simulation of plant, observer, digital control and security intervention events. Appropriate tools are system simulators and control design systems.

- C14 Supply Chain Management, SNE 11(2-3), 2001, is based on a simplified supply chain (four factories, four distributors, a group of wholesalers, and 12 products). The benchmark concentrates on discrete modelling of the process, especially of the order flow, and on implementation of different order strategies. This benchmark is a classical case study for supply chain modelling, showing also the bullwhip effect dependent on the order strategies - an interesting topic for basic education in production and logistics. The implementation does not really need a simulation system, classical recursive calculations can manage all tasks, so that the benchmark is open as educational case study in many areas.
- C15 Clearance Identification, SNE 12(2-3), 2002, is based on the dynamics of renal clearance. The benchmark puts emphasis on identification of a compartmental model for the clearance using real-word measurement protocols, and on statistical model parameter analysis based on artificial protocols. This benchmark allows to study different identification approaches for a linear dynamic system, e.g. use of sensitivity functions or use of analytical solutions, and to handle artificial data protocols for extended experiments - a useful basis for teaching of both topics, independent of the physiological background. Software tools range from compartmental modelling systems to calculation tools.

- C16 Restaurant Business Dynamics, SNE 14(1), 2004, addresses modelling, simulation and optimisation of a discrete dynamic system. The business system under examination is restaurant operating: depending on financial results (from guests living in the neighbourhood), restaurants open branches in an adequate surrounding or close down. Strategic parameters are tax rate and structural distance to new branch restaurants - to be investigated and optimised in an adequate discrete (stochastically influenced) model (process model, agent-based model, statistical analysis, event model, etc.). This benchmark addresses not only the simulation community, it is an interesting calculation task for everybody, and can be approached in various ways. Consequently, the benchmark is a basis for various education areas, and also suited for spreadsheet calculation.

- C17 Spatial Dynamics of SIR Epidemics, SNE 14 (2/3), 2004; revised SNE 25(1), 2015, investigates a classical population model for the spread of infection diseases (SIR model) and an inhomogeneous spatial approach using cellular automata. This benchmark is very suitable for educational purpose: in modelling, macroscopic and microscopic approaches can be compared, and modelling with advanced cellular automata can be studied; in implementation, discrete interventions (vaccinations) are to be managed properly for comparative investigations. And in application - spread of disease and vaccination - the benchmark is a powerful basis for learning the benefits of modelling and simulation.

- C18 Neural Networks vs. Transfer Functions, SNE 15(1), 2005,

studies alternative approaches for identification of the nonlinear dynamical relation between muscle force 
and muscle-belly thickening. Classical discrete transfer function models and as alternatives neural net models are to be compared. This benchmark may be of educational use for control modelling, and for introduction into neural net modelling and simulation software tools supporting digital control and neural net structures are recommended.

- C19 Pollution in Groundwater Flow, SNE 15(2-3), 2005, revised SNE 16(3-4), 2006,

analyses a homogeneous ground water body with a singular pollution source and with facilities for reduction of contamination, based on the transport equation. The benchmark addresses quite different modelling approaches and solution techniques, from classical discretisation methods via FEM to alternatives techniques like cellular automata, Monte-Carlo methods and Random Walk. The recent revised definition outlines the educational aspects: modelling methods for distributed systems, introduction into instationary distributed diffusion processes with comparative modelling and simulation implementation.

- C20 Complex Production System, SNE 21(3/4), 2011 is intended as a foundation for analyzing manufacturing control systems, by comparing different simulation techniques and/or control algorithms regarding complexity and dynamics. The benchmark is based on two dimensions, defining a total of twelve different scenarios that differ in their complexity and dynamic behaviour. This benchmark is a challenge for expert simulationists to test discrete process simulation systems.

- C21 State Events and Structural-dynamic Systems, SNE 26(2), 2016.

In this recent benchmark, three case studies compare modelling and implementation of state events in dynamic systems, up to structural-dynamic systems. The case studies are: the almost classical bouncing ball dynamics, a switching RLC circuit with different diode models, and the rotating pendulum with free falling phase changing dynamics from swinging to falling (and vice versa) - switching between different degrees of freedom. All three case studies are coming along with educational aspects for simulation methodology, implementation, and application. In application, the benchmark addresses mechanical engineering and electrical engineering, in methodology and implementation the benchmark deals with appropriate description and handling of hybrid and structural-dynamic systems. Depending on the case studies, the benchmark is suited for all educational levels in simulation.
- C22 Pitfalls in Discrete Event Handling, 2019/2020. This planned benchmark will discuss the proper modelling and algorithmic implementation of discrete events, emphasizing in application on flip-flops. As with recent and new benchmarks, educational aspects will be integrated from the beginning.

- CP1 Parallel Simulation Techniques, SNE 4(1), 1994, and CP2 Parallel\&Distributed Simulation, SNE16(2), 2006.

These benchmarks address mainly specialists in simulation, so the educational aspects are only minor ones.

\section{Conclusion}

The inspection of the benchmarks with respect to educational aspects shows that the benchmarks are a valuable source for use in education.

For most benchmarks a calculation tool is sufficient, so that licenses for simulation systems are not necessary. Submitted and already published educational benchmark studies and educational benchmark reports show that spreadsheet tools may be an appropriate tool for working with a benchmark. And clearly, computer numeric systems as MATLAB, Octave and Python are suitable tools and suitable alternatives to special software for system simulation.

The SNE Editorial Office invites all readers and lecturers in modelling and simulation, and lecturers in applications to make use of the ARGESIM Benchmarks, and to submit a benchmark contribution describing their use of the benchmark in education. Definitions and benchmarks solutions, benchmark reports, and benchmark studies can be found at SNE's website:

\section{www.sne-journal.org/benchmarks}

Authors are asked to submit additionally the model and/or program sources used for the benchmark contribution. Only members of EUROSIM societies can download these source files, together with the high-quality colour benchmark publications.

\section{References}

[1] Breitenecker F, Körner A, Ecker H, Pawletta T. ARGESIM Benchmarks on Modelling Approaches and Simulation Implementations - Development, Classification, and Basis for Simulation Education. SNE 29(1), March 2019, to appear.

[2] Kolozsvari Z, Lienbacher L, Perebner S, Teixeira Rego H, Bicher M, Körner A, Ecker H, Breitenecker F. Time- and Event-oriented Spreadsheet Modelling of ARGESIM Benchmark C12 'Collision Processes in Rows of Spheres'. SNE 28(4), 205 - 214, 2018.

DOI: $10.11128 /$ sne.28.bne12.10452. 